\title{
Maurer, Susanne
}

\section{Das "weibliche akademische Subjekt" - eine Forschungsnotiz}

Glaser, Edith [Hrsg.]; Andresen, Sabine [Hrsg.]: Disziplingeschichte der Erziehungswissenschaft als Geschlechtergeschichte. Opladen u.a. : Verlag Barbara Budrich 2009, S. 129-136. - (Jahrbuch Frauenund Geschlechterforschung in der Erziehungswissenschaft; 5)

\section{Quellenangabe/ Reference:}

Maurer, Susanne: Das "weibliche akademische Subjekt" - eine Forschungsnotiz - In: Glaser, Edith [Hrsg.]; Andresen, Sabine [Hrsg.]: Disziplingeschichte der Erziehungswissenschaft als Geschlechtergeschichte. Opladen u.a. : Verlag Barbara Budrich 2009, S. 129-136 - URN: urn:nbn:de:0111-opus-82398 - DOI: 10.25656/01:8239

https://nbn-resolving.org/urn:nbn:de:0111-opus-82398

https://doi.org/10.25656/01:8239

in Kooperation mit / in cooperation with:

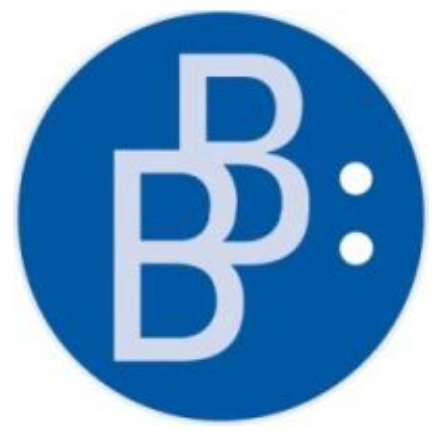

https://www.budrich.de

\section{Nutzungsbedingungen}

Gewährt wird ein nicht exklusives, nicht übertragbares, persönliches und beschränktes Recht auf Nutzung dieses Dokuments. Dieses Dokument ist ausschließlich für den persönlichen, nicht-kommerziellen Gebrauch bestimmt. Die Nutzung stellt keine Übertragung des Eigentumsrechts an diesem Dokument dar und gilt vorbehaltlich der folgenden Einschränkungen Auf sämtlichen Kopien dieses Dokuments müssen alle Urheberrechtshinweise und sonstigen Hinweise auf gesetzlichen Schutz beibehalten werden. Sie dürfen dieses Dokument nicht in irgendeiner Weise abändern, noch dürfen Sie dieses Dokument für öffentliche oder kommerzielle Zwecke vervielfältigen, öffentlich ausstellen, aufführen, vertreiben oder anderweitig nutzen.

Mit der Verwendung dieses Dokuments erkennen Sie die Nutzungsbedingungen an.

\section{Terms of use}

We grant a non-exclusive, non-transferable, individual and limited right to using this document.

This document is solely intended for your personal, non-commercial use. Use of this document does not include any transfer of property rights and it is conditional to the following limitations: All of the copies of this documents must retain all copyright information and other information regarding legal protection. You are not allowed to alter this document in any way, to copy it for public or commercial purposes, to exhibit the document in public, to perform, distribute or otherwise use the document in public.

By using this particular document, you accept the above-stated conditions of use.

\section{Kontakt / Contact:}

\section{peDOCS}

DIPF | Leibniz-Institut für Bildungsforschung und Bildungsinformation Informationszentrum (IZ) Bildung

E-Mail: pedocs@dipf.de

Internet: www.pedocs.de

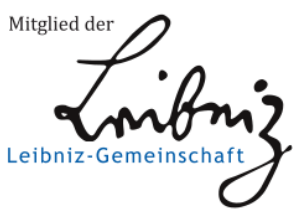


Disziplingeschichte der Erziehungswissenschaft als Geschlechtergeschichte 
Jahrbuch der

Frauen- und Geschlechterforschung

in der Erziehungswissenschaft

herausgegeben von

Sabine Andresen

Rita Casale

Edgar Forster

Edith Glaser

Vera Moser

Annedore Prengel

Barbara Rendtorff

Beirat

Birgit Althans, Berlin

Eva Borst, Mainz

Eva Breitenbach, Osnabrück

Bettina Dausien, Bielefeld/München

Isabell Diehm, Bielefeld

Hannelore Faulstich-Wieland, Hamburg

Carola Iller, Heidelberg

Marita Kampshoff, Schwäbisch Gmünd

Margret Kraul, Göttingen

Andrea Liesner, Hamburg

Susanne Maurer, Marburg

Astrid Messerschmidt, Darmstadt

Inga Pinhard, Frankfurt

Folge 5/2009 
Edith Glaser

Sabine Andresen (Hrsg.)

\section{Disziplingeschichte der Erziehungswissenschaft als Geschlechtergeschichte}

Verlag Barbara Budrich

Opladen \& Farmington Hills, MI 2009 
Bibliografische Informationen der Deutschen Nationalbibliothek

Die Deutsche Nationalbibliothek verzeichnet diese Publikation in der Deutschen

Nationalbibliografie; detaillierte bibliografische Daten sind im Internet über

http://dnb.d-nb.de abrufbar.

Gedruckt auf säurefreiem und alterungsbeständigem Papier.

Alle Rechte vorbehalten.

(C) 2009 Verlag Barbara Budrich, Opladen \& Farmington Hills, MI

www.budrich-verlag.de

\section{ISBN 978-3-86649-269-1}

Das Werk einschließlich aller seiner Teile ist urheberrechtlich geschützt. Jede Verwertung außerhalb der engen Grenzen des Urheberrechtsgesetzes ist ohne Zustimmung des Verlages unzulässig und strafbar. Das gilt insbesondere für Vervielfältigungen, Übersetzungen, Mikroverfilmungen und die Einspeicherung und Verarbeitung in elektronischen Systemen.

Satz: Susanne Albrecht-Rosenkranz, Leverkusen

Umschlaggestaltung: disegno visuelle kommunikation, Wuppertal - www.disenjo.de Druck: paper \& tinta, Warschau

Printed in Europe 
Inhalt

\section{Zur Einführung}

Edith Glaser/Sabine Andresen

Disziplingeschichte der Erziehungswissenschaft als

Geschlechtergeschichte

\section{Beiträge}

Markus Rieger-Ladich

Konturen einer machtkritischen Disziplingeschichte: Methodologische

Überlegungen und leitende Forschungsfragen zur

erziehungswissenschaftlichen Geschlechterforschung

Yvonne Ehrenspeck

Geschlechterdifferenz, Geschlechterpolarität und „Geistige

Mütterlichkeit" - systemtheoretisch beobachtet

Catrin Heite

Soziale Arbeit als Profession im Kontext geschlechterhierarchischer

Positionierungen

Astrid Kaiser

Erziehungswissenschaftliche Genderforschung in der Schulpädagogik und autonome Organisierung von Frauen und Schule

\section{Work in Progress}

Heidrun Diele/Jessika Piechocki

Sie können wie die Knaben, aber sie müssen anders erzogen werden:

August Hermann Niemeyer (1754-1828) und die Töchtererziehung

Sandra Landhäußer

Das communityorientierte Vorgehen der, Settlerinnen' von

,Hull-House“: Soziales Kapital und Perspektiven auf die

Professionalisierung Sozialer Arbeit 
Zö̈ Clark

Die Jugend ohne Jugend - Die Konstruktion der Jugendphase im Kontext ihres sozialen Ortes

Tim Köhler

Evolution ohne Frauen? Bedeutung des Geschlechtes in der

Darwinrezeption im Schulbuch um 1900

Susanne Maurer

Das ,weibliche akademische Subjekt ${ }^{\text {- }}$ eine Forschungsnotiz

\section{Rezensionen}

Karin Priem

Rezension zu: Ulrike Brunotte/Rainer Herrn (Hg.): Männlichkeiten in der Moderne. Geschlecht in den Wissenskulturen um 1900

Katja Lißmann

Rezension zu: Ulrike Gleixner / Erika Hebeisen (Hg.): Gendering Tradition. Erinnerungskultur und Geschlecht im Pietismus

Beate Ronneburger

Rezension zu: Annette Vogt: Vom Hintereingang zum Hauptportal?

Lise Meitner und ihre Kolleginnen an der Berliner Universität und in der Kaiser-Wilhelm-Gesellschaft

Margret Kraul

Rezension zu: Christa Kersting: Pädagogik im Nachkriegsdeutschland. Wissenschaftspolitik und Disziplinentwicklung 1945 bis 1955

Doreen Cerny

Rezension zu: Anne Schlüter (Hg.): Erziehungswissenschaftlerinnen in der Frauen- und Geschlechterforschung

Sabine Andresen

Rezension zu: Rita Casale/Barbara Rendtorff (Hg.): Was kommt nach der Genderforschung? Zur Zukunft der feministischen Theoriebildung ...

AutorInnenhinweise

Zum Jahrbuch Frauen- und Geschlechterforschung in der Erziehungswissenschaft

Ankündigung der nächsten Bände

Band 6

Band 7 


\title{
Das ,weibliche akademische Subjekt ${ }^{6}-$ eine Forschungsnotiz
}

\author{
Susanne Maurer
}

In diesem Beitrag wird darauf hingewiesen, dass feministisch-kritische Studien, die die unterschiedlichen Versuche, sich als ,denkende Frau' zu entwerfen und auch zu autorisieren, (werk-)biographisch rekonstruieren und in einer machtanalytischen Perspektive mit diskurs- und bewegungsgeschichtlichen Kontexten verknüpfen, noch weitgehend ausstehen. Die von ihr vorgeschlagene Forschungsperspektive richtet sich aus an den Fragen, wie sich ,weibliche Subjekte" im Feld des Akademischen in der Vergangenheit tatsächlich situieren konnten, welche Strategien der Teilhabe sie vor dem Hintergrund ihres ,systematischen Ausschlusses' entwickelten und wie sie ,das Akademische' selbst reflektierten. Maurer erinnert in diesem Zusammenhang an einen Essay von Marianne Schuller (1984) zum Verhältnis von ,weiblicher Intellektualität' und Macht und nimmt in einem zweiten Schritt beispielhaft auf eine der ersten Pädagogik-Professorinnen (Elisabeth Blochmann, 1892-1972) und deren Selbstrealisierung als Wissenschaftlerin und Hochschullehrerin Bezug.

\section{The `female academic subject` - a research note}

Susanne Maurer emphasizes in her article the clear lack of critical feminist studies which reconstruct the diverse attempts of conceptualizing oneself as a "qualified" female intellectual. Her suggested research perspective focuses on questions such as, how 'female subjects' of the past could situate themselves within the academic power-field; which strategies of participation did they develop being confronted with the systematic exclusion of women, and how did they reflect the academic sphere itself? Maurer suggests a perspective which connects a power-analytical approach inspired by Michel Foucault with exegetical, biographical, discourse-analytical and historical perspectives, taking the collective dimension of feminist movements as an historical context also into account. In her short contribution Maurer recalls a theoretical essay on 'female intellectuality' by Marianne Schuller (1984) and refers to one of the first female professors of educational science as an example, Elisabeth Blochmann (1892-1972). 
„Und geblieben ist der prekäre Status [...] Keine Normalität also [...] Was das bedeutet, [...] spüre ich täglich am kulturellen Leib: beständiger Abfluss von Befassungsenergie. Beständige Positionierungs-, Sozial- und Verhaltensarbeit in einer Institution mit frauenundurchlässiger Hierarchie. Beständiges Austarieren der Aporien von Berufsrolle und Geschlechtsrolle zwischen Stillstellen, Suspendieren und Zulassen von Weiblichkeit. Maskierung-Mimikry-Parodie - Subversion: täglicher Affentanz. Keine Normalität. Unter den Bedingungen von social grace und Lächelzwang zwanzig Jahre Aporie-Verwaltung [...] Wie jede andere, die von hier aus spricht, bin ich Überlebende des akademischen Frauensterbens. "

(Hassauer 1994, S. 34f.)

Diesen Befund formuliert Friederike Hassauer im Rahmen ihrer Antrittsvorlesung, in der sie den ,systematischen Ort weiblicher Subjekte im Feld des Akademischen' historisch und theoretisch rekonstruiert. Sie stellt sich damit in eine interessante Tradition, denn vor ihr haben etwa Michel Foucault oder Pierre Bourdieu das Genre der Antrittsvorlesung genutzt, um entweder ,die Ordnung des Diskurses“ (Foucault 1991) oder den Ritus der Antrittsvorlesung im Kontext der symbolischen Machtwirkungen des Wissenschafts,Spiels‘ (vgl. Bourdieu 1985) zu analysieren. Doch die Ungleichheit, die Hassauer zum Thema macht, ist eine noch andere als diejenige, die Bourdieu reflektiert: Frauen treffen nicht nur auf eine männerbündisch und homosozial strukturierte Organisation des ,Wissenschaftskörpers` (vgl. Hassauer 1994, S. 33) - das Konzept von Wissenschaft selbst entwickelte sich historisch und kulturell als ,das Andere der Weiblichkeit'.

Feministisch-kritische Studien, die vor diesem Hintergrund die unterschiedlichen Versuche, sich als, denkende Frau' zu entwerfen und auch zu autorisieren, (werk-)biographisch rekonstruieren und in einer machtanalytischen Perspektive mit diskurs- und bewegungsgeschichtlichen Kontexten verknüpfen, stehen noch weitgehend aus. ${ }^{1}$ Hier ergibt sich - gerade im Hinblick auf die Konzeption weiblicher Intellektualität als ,geistige“ oder gar „,pädagogische“ Mütterlichkeit (vgl. hierzu Bührmann 2004) - ein für die Erziehungswissenschaft höchst interessantes Forschungsfeld, das die Disziplingeschichte als Geschlechtergeschichte noch weiter erhellen kann.

Mit diesem kurzen Beitrag möchte ich eine entsprechende Forschungsperspektive zumindest andeuten. Sie richtet sich an den Fragen aus, wie sich ,weibliche Subjekte' im Feld des Akademischen in der Vergangenheit tatsächlich situieren konnten, welche Strategien der Teilhabe sie vor dem Hintergrund ihres ,systematischen Ausschlusses' entwickelten und wie sie ,das Akademische' selbst reflektierten. ${ }^{2}$ In diesem Zusammenhang werde ich zu-

1 Vgl. zu einem Versuch, mit Hilfe des Konzepts der „dissidenten Teilhabe“ insbesondere die Bewegungen feministischer Wissenschaftlerinnen im ,System Universität' zu reflektieren, Maurer 1999.

2 So wurde etwa im Kontext der Frauenbewegungen um 1900 die Frage der ,intellektuellen Grenzlinien“ (Lange 1897) im ,Geschlechterverhältnis“, in der gesellschaftlichen und sym- 
nächst an einen Text von Marianne Schuller zum Verhältnis von ,weiblicher Intellektualität‘ und Macht erinnern (vgl. Schuller 1984), an den heutige Analysen meines Erachtens produktiv anschließen können. Im zweiten Schritt nehme ich auf Elisabeth Blochmanns Überlegungen zur ,akademischen Lebensform' Bezug und frage danach, wie sie selbst sich als ,weibliche Akademikerin' realisiert hat.

Elisabeth Blochmann (1892-1972) war die erste Pädagogik-Professorin in Westdeutschland nach 1945. ${ }^{3}$ Im Alter von 60 Jahren erhielt sie einen zunächst außerordentlichen - Lehrstuhl für Pädagogik in Marburg. Außer ihr gab es mit der Literaturwissenschaftlerin Luise Berthold damals nur noch eine einzige andere Professorin an der dortigen Universität. Blochmann wirkte als Direktorin des Marburger Pädagogischen Seminars und entwickelte die geisteswissenschaftliche Pädagogik in einer Variante weiter, die insbesondere auch die Mädchen- und Frauenbildung in den Blick nahm (vgl. Müller 1993a, S. 41). Schon Ende der 1920er Jahre hatte sie diverse Angebote für Professuren erhalten - so etwa von den Pädagogischen Akademien in Halle und Kassel sowie von der Universität Jena (vgl. ebd., S. 49). Die reformpädagogisch inspirierte Situation in Halle zog Blochmann offenbar an - sie gestaltete den Aufbau der Pädagogischen Akademie dort von Anfang an mit, lehrte Sozialpädagogik und bildete Volksschullehrerinnen und -lehrer auch im Bereich der Fürsorge aus. Mit ihren Kollegen Adolf Reichwein und Martin Rang verband sie die Orientierung am pädagogischen Handeln als gemeinsames Moment im - gesellschaftlich gerahmten und bedingten - Alltag von Lehrenden und Lernenden (vgl. ebd., S. 50).

1933 musste Elisabeth Blochmann - wie noch neun andere ihrer Kollegen und Kolleginnen - die Pädagogische Akademie verlassen, weil ihre Mutter Jüdin war (vgl. auch hierzu ebd., S. 52f.). 1934 emigrierte sie nach England, und erhielt dort die Möglichkeit, an der University of Oxford zu arbeiten. Erst 1952 kehrte sie ganz nach Deutschland zurück.

„Die Ambivalenz der Weiblichkeit als ,Rest` der Intellektualisierungsprozeduren ist ... im Machtspiel nicht ganz aufgezehrt. Sie bleibt, unbestimmter Ort einer Unruhe, einer Erfahrung von Differenz, als Heterogenität wirksam. Sie kommt mit keiner verschriebenen Identifikation ganz zu Rande, sie ist überschüssig. Und darin, in dieser flottierenden Bewegung, auch widerständig." (Schuller 1984, S. 17)

bolischen Geschlechterordnung, verhandelt. Auch die Neuen Frauenbewegungen seit Ende der 1960er Jahre diskutierten in vielfältiger Weise das Verhältnis von ,Wissenschaft und Weiblichkeit‘ (vgl. dazu Maurer 1996).

3 Noch vor Elisabeth Blochmann war Mathilde Vaerting (1884-1977) die erste Professorin, die in Deutschland das Fach Pädagogik vertrat (vgl. Kraul 1987; Müller 1993b). Seit Oktober 1923 - das erste Jahr, in dem Frauen überhaupt als Ordinaria berufen werden konnten lehrte und forschte sie als ordentliche Professorin für Soziologie und Erziehungswissenschaft an der Thüringischen Landesuniversität Jena. - Vgl. zur komplizierten und spezifischen Situierung von Vaerting im akademischen Feld Wobbe 1998b, die Vaerting für die Disziplingeschichte der Soziologie rezipiert. 
In ihrem Aufsatz „Vergabe des Wissens“ arbeitet Marianne Schuller mit Bezug auf Foucaults Analytik der Macht, auf Sigmund Freud und Jacques Lacan heraus, wie gerade am ,Fall“ weiblicher Intellektueller die „Ausbildung und Anerkennungsmechanismen [von Intellektualität; Sus. M.] selbst als Effekt und Transfer von Macht rekonstruierbar" werden (ebd., S. 15). Intellektualität stelle, so Schuller, gegenüber „der patriarchalen Weiblichkeitskodierung etwas Fremdes dar“ (ebd.). Die angesichts weiblicher Intellektueller entstehende Irritation werde nun in der Weise gestaltet, dass Intellektualität im ,Fall der Frau' nicht als (selbst-)schöpferischer Akt oder auch (Selbst-)Bildungsprozess, sondern als „Gabe“ (ebda, S. 16) gedacht werde. Die ge- oder verabreichte ,Gabe Intellektualität“ stehe der Frau „von geschlechtswegen“ eigentlich gar nicht zu und werde deshalb zu einem prekären Gut, womöglich gar zur Schuld. ${ }^{4}$

Um die solchermaßen gefährdete, bedrohte, instabile Lage abzusichern, werden von ,weiblichen Intellektuellen' (von Frauen also, die Intellektualität ,begehren', an ihr teilhaben wollen) selbst Strategien entwickelt, die zu einer aktiven Unterstützung und Reproduktion derjenigen Mechanismen noch beitragen, mittels derer ihnen der Intellektuellen-Status vorenthalten wird. Die damit angezeigte „dilemmatische Situation“ führt allerdings auch „Versuche von Frauen, sich im Einspruch gegen die sie selbst formierende Macht als Intellektuelle hervorzubringen“ (ebd., S. 17) mit sich: „Von der Problemlage her müssen diese Versuche, die sich auf politisch-praktischen und theoretischen Feldern in unterschiedlicher Weise abspielen, radikal sein, weil sie ... an eine Wurzel der Machtkonstitution selber rühren" (ebd.).

Frauen haben also ,allen Grund, intellektuelle Widerstandsformen im Zuge radikaler Machtkritik zu entwickeln“ (ebd.). Von daher erklärt sich auch die Attraktion vernunft- und wissenschaftskritischer Perspektiven für die feministische Theorieentwicklung (vgl. hierzu u.a. Maurer 1996). Die notwendige "Störung der machtrelevanten Anerkennungssysteme“ (Schuller 1984, S. 18) kann sich, so deutet Schuller an, gerade auf dem Wege einer dekonstruktiven und machtanalytischen Rekonstruktion von Wissenschaft als „,äterlichem Wissen“" und „massive[r] Form von Macht“" einstellen:

„Das heißt auch, dass weibliche Intellektualität sich nicht diesseits oder jenseits des ,väterlichen Wissens' und der Macht, sondern nur in deren Subversion entfalten kann: indem sie sich nicht zufrieden gibt mit den innerhalb der Szenen oder institutionell vorgesehen Plätzen, auch nicht mit dem der Kritik, sondern die Zuweisung und Platzverteilung als Machtfunktion selbst

4 Susanne Omran verdeutlicht die Problematik des Schuldhaften am Beispiel der österreichisch-tschechischen Schriftstellerin und Frauenrechtlerin Grete Meisel-Hess (vgl. Omran 1999). - Am Beispiel von Marianne Weber ließe sich der Weg einer Frau, die trotz gelegentlich anderslautender Empfehlungen des eigenen Partners (Max Weber), weiterhin selbständig wissenschaftlich arbeitet, in sehr interessanter Weise reflektieren; vgl. hierzu nicht zuletzt Weber selbst (Weber 1919), sowie Bührmann 2004 und Wobbe 1998a. 
in Frage stellt; indem sie sich nicht mit der Gabe identifiziert und nicht mit dem darüber Erreichten. Vielmehr entzündet sich ,weibliche Intellektualität‘ [...] an den ,Resten', die die Gesten und Rituale der Macht erzeugen. In diesem Sinne ist sie deren Negativ und Störung. Der Prozess der Störung aber muss sich auch auf sie selbst beziehen: ihr eigner Mangel taucht nicht als eine schleunigst zu substituierende Schwäche, sondern als ein produktives Moment auf. Er macht sie neugierig. Begierde mischt sich in die Intellektualität." (ebd., S. 19)

An dieser Stelle wird im Text eine „Zwischenbilanz“ formuliert - die Autorin sieht in den (1984!) gegenwärtigen Praktiken intellektueller Frauenszenen innerhalb wie außerhalb der Institutionen eher ein Scheitern des von ihr selbst skizzierten Projektes, denn einen ernsthaften Versuch. Einen Grund hierfür macht sie in einer allzu schnellen Geste der Repräsentation aus: die (historisch-kulturell-konzeptionell eigentlich ,unmögliche') ,weibliche Intellektuelle' setze sich als , andere Intellektuelle' einfach an die leere Stelle der fehlenden kulturellen Muster für weibliche Intellektualität, und mache damit deren Nichtvorhandensein unsichtbar. Damit ,aber geht genau die mit der Ambivalenz der weiblichen Position verbundene theoretische und politische Sprengkraft verloren“ (ebd., S. 20). Wie sich dies zudem, insbesondere in den von Gleichheits-Phantasien, -Wünschen und auch -Dogmen durchzogenen frauenbewegten Kreisen (zumindest der 1980er Jahre), mit neuen , weiblichen Machtformen“ verknüpft, macht Schuller ebenfalls deutlich: „Im Schatten imaginärer Gleichheit hinterlässt sie [die Macht; Sus. M.], fast unangreifbar, ihre hierarchisierenden Spuren. Und in dem Maße, in dem sie nicht zu fassen ist, sind die Individuen ihren Effekten ausgesetzt: Konkurrenzkämpfe, Anerkennungsstrategien und der Zwang zu gepanzerter Selbstinszenierung sind die Folgen“" (ebd., S. 21).

„Die männliche Studentenschaft und die Dozentenschaft hat noch kaum realisiert, welcher Gewinn darin liegt, dass die akademische Welt keine rein männliche mehr ist, sondern eine im vollen Sinn menschliche - sein sollte." (Blochmann 1963, S. 13)

Im Jahr 1963 hält Elisabeth Blochmann im Radio Bremen einen Vortrag zur „akademischen Lebensform“, der noch im selben Jahr in der von ihr selbst mit herausgegebenen Zeitschrift „Die Neue Sammlung“ erscheint (vgl. Blochmann 1963). In diesem Vortrag macht sie die bildende Wirkung des ,akademischen Lebens“ zum Thema, die Lebens- und Beziehungsverhältnisse von Studierenden und Lehrenden, auch und gerade im internationalen Kontrast. Die Frage der damit sich ebenfalls verbindenden Geschlechterverhältnisse klingt leise an in diesem Text - gegen Ende der Ausführungen geht Blochmann auch explizit auf die weiblichen Studierenden ein, auf die Frage, was das Studium in ihrem Lebenszusammenhang bedeutet, und auf die Frage, was ihre Anwesenheit für die Universität bedeutet - oder doch bedeuten könnte.

Ihren Text 45 Jahre später lesend, verblüfft mich (oder auch nicht), welche zentralen und existentiellen Fragen - für die Universität als Institution 
und Komplex, für die darin studierend wie lehrend Tätigen und sich Bewegenden, für das Geistesleben der Gesellschaft insgesamt - hier mit großer Klarheit angesprochen und reflektiert werden.

Elisabeth Blochmann ist die erste Professorin für Erziehungswissenschaft in Marburg und die erste neuberufene Professorin für Erziehungswissenschaft in der Bundesrepublik Deutschland überhaupt; die Entwicklung der Universität kommentiert sie vor dem Hintergrund ihrer eigenen Position als weibliche Wissenschaftlerin in einem männlich dominierten Feld. Dies macht sie in dem hier kurz vorgestellten Text allerdings nicht explizit zum Thema. Dennoch mag ihre Akzentuierung des „akademischen Lebens“ als Lebenszusammenhang etwas mit ihrer spezifischen Position im Gefüge der Universität zu tun haben: Mit Bezug auf Johann Gottlieb Fichte, Friedrich Schleiermacher und Herman Nohl, aber auch auf Kollegen in England und den USA, rekonstruiert (eher: moniert) sie die Beziehungsdimension, und insbesondere die Praxis und Kultur der Geselligkeit im modernen universitären Geschehen.

Gleichzeitig erinnert sie an die zentrale gesellschaftsgeschichtliche Funktion der (deutschen) Universität: „Bildung wurde verstanden als das große Mittel, den jungen Menschen bürgerlicher Herkunft aus der kleinbürgerlichen Enge und der Subalternität, in der er befangen war, zu befreien und in ihm eine neue Lebenshaltung zu erzeugen. [...] so bekam die Universität dadurch, dass sie einem großen Teil der Jugend aus dem Bürgertum die Befreiung des Geistes und damit eine eigene Ehre - in der Intention jedenfalls - ermöglichte, eine der wichtigsten Funktionen im Prozess der sozialen Umschichtung, die mit der Befreiung des Bürgertums begann“ (ebd., S. 8).

Weit davon entfernt, die Geschichte und Gegenwart der deutschen Universität zu idealisieren - Blochmann macht in ihrer Rede ebenfalls deutlich, dass hier dem Nationalsozialismus wenig entgegengesetzt wurde -, besteht sie doch auf der potentiell bildenden Kraft einer - anderen, erst noch zu entwickelnden - Universität, die viel mit Integrität, Freiwilligkeit und Freiheit zu tun hat: „Neben [respektvollem; Sus. M.] Umgangsstil und Freiwilligkeit aber gibt es noch ein weiteres wichtiges Moment, das sich freilich mit dem ersten berührt. [...] Die Education, die einem eine der alten Universitäten [in England; Sus. M.] schenkt, besteht außer in der Geistesbildung durch die Arbeit am Gegenstand des Studiums in der Teilhabe an der Totalität des akademischen Lebens, des geistigen und des geselligen, auch wohl des sportlichen" (ebd., S. 11).

Einige Schlüsselsätze, die sich auf die Präsenz und Erfahrung von Frauen in der Universität beziehen ${ }^{5}$, finden sich im Zusammenhang mit Blochmanns

5 Helmut-Gerhard Müller verweist in seinem Portrait der Professorin Blochmann auf den Umstand, dass sie sich als ,Privatperson' kaum zu erkennen gegeben habe. Das könne nicht zuletzt mit der - zumindest anfangs - recht feindseligen Haltung ihrer Kollegen in Marburg zusammenhängen (vgl. Müller 1993a, S. 41 und 58). - Ihre ehemaligen Marburger Studen- 
Betonung des Geselligen (insbesondere auch unter den Studierenden), im Unterschied zur ,,andere ausschließende[n] Zweisamkeit“, in die sich viele ,zu früh“ hineinflüchteten (vgl. ebd., S. 13). Ob das Studium „,zum Lebensberuf und zur Erfüllung ihrer Tage" (ebd.) führen werde, sei für Frauen unsicher und ungewiss. Daraus ergebe sich ein ,innerer Vorbehalt“, der das Leben einer Studentin sehr belasten könne (ebd.). Dieser Vorbehalt „kann aber, richtig verstanden, auch ein Gewinn sein, weil er eine innere Distanz zu dem Zwang des Tages ermöglicht und damit eine geistige Freiheit auch dem Dogma des Lehrers und dem Druck der Sache gegenüber" (ebd.).

Mit dieser Überlegung reiht sich Blochmann in gewisser Weise ein in Reflexionen, die aus der spezifischen - oft randständigen - Situiertheit weiblicher Erkenntnissubjekte eine zumindest potentiell unabhängigere Erkenntnisperspektive rekonstruieren.

Elisabeth Blochmann jedenfalls, die bei vielen spannenden Denkern ihrer Zeit (wie Wilhelm Rein, Georg Simmel, Paul Natorp, Herman Nohl) studiert hat, erweist sich als sehr eigenständige Wissenschaftlerin, die auch mit dem für sie so bedeutsamen Nohl eine ebenbürtige akademische Freundschaft entwickelt. Eine „Gefolgschaftstreue“ lehnt sie dezidiert ab und kritisiert in dieser Hinsicht z.B. Eduard Spranger, der (hier: in Bezug auf Friedrich Fröbel) „hero-worship“ betreibe und damit eine „,Erneuerung“ des autoritätsgläubigen ,Geistes“ “ begünstige (Blochmann 1953, zit. nach Müller 1993a, S. 64).

Interessant erscheint mir schließlich noch ein anderer Hinweis aus Helmut-Gerhard Müllers Studie zur akademischen Biographie der Marburger Pädagogin: Elisabeth Blochmann hatte über ihre Freundin Elfride Petri den Philosophen Martin Heidegger kennengelernt. Seinen Empfehlungen in Bezug auf mögliche Themenschwerpunkte für eine Dissertation folgte Blochmann allerdings nicht. Heideggers Fragment „Sein und Zeit“, das jener ihr im Juni 1924 zugeschickt hatte, hat sie vermutlich nie intensiver gelesen, auch andere - mit persönlichen Widmungen an Blochmann versehene ${ }^{6}-$ Werke $^{-}$ Heideggers in ihrem Nachlass wirken wie unberührt (vgl. hierzu Müller 1993a, besonders S. 46ff. und S. 68, FN 22).

Wenn die junge Elisabeth Blochmann sich diesen Heidegger'schen Literatur,gaben' kaum widmete, so hat dies ja vielleicht nicht zuletzt auch damit etwas zu tun, dass hier eine nicht ,von Mannes Gnaden denken', sondern ihre eigenen Fragen und Themen entwickeln wollte? Gerade in den ,beiläufigen“ Hinweisen finden sich meines Erachtens die Spuren der Eigen-Bewegung im vorgezeichneten Feld.

tinnen erinnern sich an Blochmann als ,strenge Professorin', die sich - im Unterschied zu ihrer Kollegin Luise Berthold - stets ,weiblich-elegant' gekleidet habe.

6 Heidegger sprach Blochmann in seinen Briefen im Übrigen explizit als ,weibliche Forscherin' an (vgl. Müller 1993a, S. 46). 


\section{Literatur}

Blochmann, Elisabeth (1963): Die akademische Lebensform. In: Neue Sammlung. 3. Jg., S. 7-14

Bourdieu, Pierre (1985): Sozialer Raum und „Klassen“. Leçon sur la leçon. Zwei Vorlesungen, Frankfurt a. M.

Bührmann, Andrea (2004): Der Kampf um weibliche Individualität. Zur Transformation moderner Subjektivierungsweisen in Deutschland um 1900, Münster

Foucault, Michel (1991): Die Ordnung des Diskurses, Frankfurt a. M.

Kraul, Margret (1987): Geschlechtscharakter und Pädagogik: Mathilde Vaerting (1884-1977). In: Zeitschrift für Pädagogik. 33. Jg., S. 474-489

Lange, Helene (1897): Intellektuelle Grenzlinien zwischen Mann und Frau. In: Die Frau. Monatsschrift für das gesamte Frauenleben unserer Zeit. 4. Jg., Heft 6, S. 321-334

Maurer, Susanne (1999): Zwischen „freiwilliger Assimilation“ und „dissidenter Teilhabe“. Feministische Wissenschaftlerinnen im dominanten System Universität, in: Treptow, Rainer/Hörster, Reinhard (Hg.): Sozialpädagogische Integration. Entwicklungsperspektiven und Konfliktlinien. Weinheim und München 1999, S. 223-235

Maurer, Susanne (1996): Zwischen Zuschreibung und Selbstgestaltung. Feministische Identitätspolitiken im Kräftefeld von Kritik, Norm und Utopie, Tübingen 1996

Müller, Helmut-Gerhard (1993a): Elisabeth Blochmann (1892-1972) in Marburg an der Lahn, in: Frauen in Marburg. Ein Lauf- und Lesebuch. Hrsg. vom DGB Kreis Marburg-Biedenkopf in Zusammenarbeit mit der Frauenbeauftragten der Stadt Marburg. Marburg, S. 41-70

Müller, Helmut-Gerhard (1993b): Mathilde Vaerting (1884-1977), in: Frauen in Marburg. Ein Lauf- und Lesebuch. Hrsg. vom DGB Kreis Marburg-Biedenkopf in Zusammenarbeit mit der Frauenbeauftragten der Stadt Marburg, Marburg, S. 7387

Omran, Susanne (1999): Weib und Geist um 1900. Intellekt, Rasse und Instinkt in den Schriften von Grete Meisel-Hess, in: Die Philosophin 19 (,Intellektualität und Weiblichkeit"), 10. Jg., S. 11-35

Schuller, Marianne (1984): Vergabe des Wissens. Notizen zum Verhältnis von „,weiblicher Intellektualität“ und Macht, in: Konkursbuch 12 („Frauen Macht“), hrsg. von Claudia Gehrke, Gerburg Treusch-Dieter und Brigitte Wartmann, Tübingen 1984, S. 13-21

Weber, Marianne (1919): Frauenfragen und Frauengedanken. Gesammelte Aufsätze, Tübingen 1919

Wobbe, Theresa (1998a): Marianne Weber (1870-1954). Ein anderes Labor der Moderne, in: Frauen in der Soziologie. Neun Portraits. Hrsg. von Claudia Honegger und Theresa Wobbe. München, S. 153-177

Wobbe, Theresa (1998b): Mathilde Vaerting (18840-1977). Die Macht des Unterschieds, in: Frauen in der Soziologie. Neun Portraits. Hrsg. von Claudia Honegger und Theresa Wobbe. München, S. 178-202 